\title{
EDUCAÇÃO EM SAÚDE E SEXUALIDADE: EXPERIÊNCIA COM ADOLESCENTES DO PROGRAMA DE ERRADICAÇÃO DO TRABALHO INFANTIL
}

\author{
HEALTH EDUCATION AND SEXUALITY: EXPERIENCE WITH TEENAGERS IN THE CHILD LABOR \\ ERADICATION PROGRAM \\ EDUCACIÓN EN SALUD Y SEXUALIDAD: EXPERIENCIA CON ADOLESCENTES EN EL PROGRAMA DE \\ ERRADICACIÓN DEL TRABAJO INFANTIL \\ Carla Patrícia Vieira de Miranda \\ Raquel Martins Mororó \\ Suyanne Maria Brito de Souza Rocha \\ Tiara Bruna Teixeira Teodósio \\ Maria Adelane Monteiro da Silva \\ Rebeca Sales Viana
}

\section{RESUMO}

$E$

ste artigo relata a experiência de acadêmicas de graduação em Enfermagem da Universidade Estadual Vale do Acaraú (UVA) no - desenvolvimento de ações de educação em saúde sobre sexualidade com adolescentes do Programa de Erradicação do Trabalho Infantil (Peti) de Sobral (CE). Tais momentos foram conduzidos empregando as técnicas de observação participante, grupo focal e sociodrama. Para tanto, foram utilizados como recursos: diário de campo, dinâmicas de grupo, jogos didáticos e atividades lúdicas. Os adolescentes participaram dos encontros discutindo a temática e procurando esclarecer suas dúvidas.

Palavras-chave: Adolescência; Sexualidade; Educação em Saúde.

\footnotetext{
1. Aluna de graduação em Enfermagem na Universidade Estadual Vale do Acaraú (UVA). Sobral (CE), Brasil.

2. Aluna de graduação em Enfermagem na UVA. Sobral (CE), Brasil.

3. Aluna de graduação em Enfermagem na UVA. Sobral (CE), Brasil.

4. Aluna de graduação em Enfermagem na UVA. Sobral (CE), Brasil.

5. Enfermeira. Pós-Doutora em Enfermagem pela Universidade Federal do Ceará (UFC). Professora no curso de Enfermagem da UVA. Sobral (CE), Brasil.

6. Cirurgiã-dentista. Mestre em Gestão Pública pela UVA. Professora no curso de Enfermagem da UVA. Sobral (CE), Brasil.
} 


\section{ABSTRACT}

This article reports the experience of undergraduate Nursing students from the Ceara State University Acaraú Valley (UVA) in developing health education actions on sexuality with teenagers attending the Brazilian Child Labor Eradication Program (PETI) in Sobral, Ceará, Brazil. These moments were conducted using the techniques of participant observation, focus group, and sociodrama. To do this, the resources used were: field diary, group dynamics, didactic games, and ludic activities. Teenagers participated in the meetings by discussing the theme and trying to resolve their doubts.

Keywords: Adolescence; Sexuality; Health Education.

\section{RESUMEN}

Este artículo reporta la experiencia de estudiantes de pregrado en Enfermería de la Universidad del Estado de Ceará Valle de Acaraú (UVA) en el desarrollo de acciones de educación en salud acerca de sexualidad con adolescentes del Programa de Erradicación del Trabajo Infantil Brasileño (PETI) en Sobral, Ceará, Brasil. Estos momentos se llevaron a cabo utilizando las técnicas de observación participante, grupo focal y sociodrama. Para hacer esto, los recursos utilizados fueron: diario de campo, dinámica de grupo, juegos didácticos y actividades lúdicas. Los adolescentes participaron en los encuentros discutiendo el tema y tratando de resolver sus dudas.

Palabras clave: Adolescencia; Sexualidad; Educación en Salud.

\section{INTRODUÇÃO}

A adolescência se caracteriza como um período marcado por instabilidades psicológicas, culturais, fisiológicas e sociais. Nesse cenário, diariamente, os adolescentes são bombardeados por excesso de informações, muitas vezes repassadas de modo confuso e desconexo, o que resulta em uma contextualização desordenada.

A sexualidade se insere nesse processo, sobretudo, como um elemento estruturador da identidade do adolescente. No entanto, muitos pais têm dificuldade, e até vergonha, de falar sobre sexo com seus filhos, seja devido à educação e aos exemplos que vivenciaram ou pelo desconhecimento acerca do uso correto de preservativos e contraceptivos ${ }^{1}$.

Nesse sentido, mostra-se pertinente trabalhar ações de educação em saúde com adolescentes, levantando seus focos de interesse e considerando nessas estratégias as fases de desenvolvimento, as diferenças culturais e as crenças religiosas dos participantes.

No que concerne à atuação do profissional da saúde, a abordagem junto ao adolescente deve diferir daquela voltada ao adulto, pois além de conhecimento técnico-científico, o profissional também precisa de habilidade para promover uma participação ativa do jovem².

Portanto, a ação educativa em saúde constitui processo dinâmico que tem por objetivo a capacitação de indivíduos e/ou grupos em busca da melhoria das condições de saúde da população. Trata-se de um processo que estimula o diálogo, a indagação, a reflexão, o questionamento e a ação partilhada ${ }^{3}$.
As ações descritas foram realizadas com adolescentes do Programa de Erradicação do Trabalho Infantil (Peti) “Betesda", no Centro de Referência de Assistência Social (Cras), em Sobral (CE), em virtude dessa unidade de atenção à saúde se relacionar com projetos socioeducativos de grande impacto nesse município.

A escolha de adotar como objeto de estudo a sexualidade do adolescente no âmbito do Peti decorreu da identificação de lacunas de conhecimento, a partir de levantamento inicial realizado com esse público-alvo, por meio de uma oficina na qual foram abordados eixos relativos à temática. Foram observadas as dificuldades dos adolescentes participantes do estudo para entender as questões e os mitos que envolvem a abordagem da sexualidade, embora o desenvolvimento de ações por parte dos profissionais da saúde atenda aos anseios dessa clientela no serviço em análise. Portanto, nessa perspectiva, sentimos necessidade de fortalecer ações de educação em saúde com esse público-alvo, voltadas à discussão da sexualidade.

0 Peti atende a uma demanda de crianças e adolescentes na etária de 6 a 15 anos, articulando um conjunto de ações que visa à retirada de crianças e adolescentes de até 16 anos de práticas de trabalho infantil. A proposta envolve reconhecer a criança e o adolescente como sujeitos de direitos, protegê-los contra as diversas formas de exploração do trabalho e contribuir com seu desenvolvimento integral ${ }^{4}$.

Nesse cenário, surgem as seguintes questões:

- Como os adolescentes experienciam a sexualidade?

- Quais são suas angústias e suas dúvidas diante da temática? 
- Como o aprendizado construído em encontros de educação em saúde pode contribuir para vivenciar melhor a sexualidade nessa etapa da vida?

Este artigo descreve as ações de educação em saúde sobre sexualidade realizadas por acadêmicas de Enfermagem com adolescentes do Peti.

\section{METODOLOGIA}

Trata-se de relato de experiência realizada em setembro e outubro de 2012 em Sobral.

A experiência foi desenvolvida em 3 encontros, na sede do Peti no município, que atendia uma demanda de 98 adolescentes. Participaram dos momentos os indivíduos com idades entre 12 e 15 anos.

Cada momento teve duração de 3 horas, no turno vespertino, contando com a participação de cerca de 15 adolescentes. 0 Quadro 1 detalha os encontros e os instrumentos de registro utilizados para a coleta de dados.

Quadro 1 - Técnicas e instrumentos utilizados na condução de cada encontro

\begin{tabular}{lcc}
\hline & $\mathbf{1}^{\mathbf{0}}$ momento & $\mathbf{2}^{\circ}$ momento \\
\hline $\begin{array}{l}\text { Metodologia } \\
\text { Observação participante }\end{array}$ & Grupo focal \\
$\begin{array}{l}\text { Instrumentos de } \\
\text { registros utilizados para }\end{array}$ & Diário de campo & Fotografias \\
\hline
\end{tabular}

Fonte: Elaborado pelas autoras.

Para a análise e discussão dos resultados, recorremos à técnica de análise de conteúdo, de acordo com a proposta de Minayo ${ }^{5}$, junto com os pressupostos da técnica sociodramática.

Este estudo foi norteado pela Resolução do Conselho Nacional de Saúde (CNS) n. 466/2012.

\section{RESULTADOS}

\section{Descrevendo os encontros}

0s encontros ocorreram em 3 momentos. 0 primeiro deles visou a conhecer os componentes do grupo, bem como levantar o comportamento dos adolescentes diante das atividades propostas, além de possibilitar a familiarização com as expressões verbais e não verbais e com a interação do grupo.

A técnica de observação participante consiste na inserção do pesquisador no interior do/a grupo/realidade observada, tornando-se parte dele/a, ao interagindo com os sujeitos e partilhar seu cotidiano para identificar o que significa encontrar-se naquela situaçã $0^{6}$.

No segundo momento, a técnica do grupo focal foi utilizada para levantar as principais dúvidas dos adolescentes sobre o tema sexualidade e despertar seu interesse na busca do autoconceito e da interação grupal.

0 grupo foi dividido em 3 subgrupos de 4 adolescentes. Em seguida, realizou-se a dinâmica denominada "o semáforo", na qual deveriam classificar perguntas relativas à temática sexualidade como fácil entendimento (sinal verde), médio entendimento (sinal amarelo) e difícil entendimento (sinal vermelho), abordando estes aspectos: doenças sexualmente transmissiveis (DST), paquera, gravidez na adolescência, masturbação, sexo e preservativos e contraceptivos.

os participantes definiram como sinal vermelho as questões sobre DST, contraceptivos e masturbação. Apresentaram como sinal amarelo os assuntos sobre sexo e preservativos. E o sinal verde se referiu a questões sobre paquera e gravidez na adolescência.

Após a realização dessa dinâmica, foi proporcionado um momento para discussão das principais dúvidas relativas aos aspectos em foco.

Os encontros grupais possibilitam aos participantes explorar seus pontos de vista, a partir de reflexões sobre determinado fenômeno social, com base em seu próprio vocabulário, gerando suas próprias perguntas e buscando respostas pertinentes à questão em análise. Desse modo, o grupo focal pode atingir um nível reflexivo que outras técnicas não conseguem alcançar, revelando dimensões de entendimento que, frequentemente, permanecem inexploradas pelas técnicas convencionais de coleta de dados ${ }^{7}$.

Em acordo mútuo com a observação participante e o grupo focal, no terceiro momento foi aplicada a técnica do sociodrama de acordo com a proposta de Jacob Levy Moreno7, para estimular os aspectos da espontaneidade e oferecer aos participantes mapeamentos dos papéis exercidos no próprio grupo e no meio social.

Tal encontro se iniciou com a elaboração, pelos próprios 
adolescentes, de 2 temas emergidos do interesse de discussão sobre o assunto, com os seguintes tópicos:

"Ele só usa camisinha com uma garota séria, com outras nem liga"; e

"Meu filho se masturba todo dia."

0 sociodrama é um método de pesquisa interventiva, que busca compreender os processos grupais e intervir em uma de suas situações-problema, por meio da ação/comunicação das pessoas. Como todas as abordagens de Jacob Levy Moreno, trata-se de um método de ação que movimenta e permite a concretização de transformações a partir da consciência e da verdade desvelada pelo próprio protagonista, que, no caso, é o grupo ${ }^{8}$.

A partir da participação ativa dos adolescentes nos debates, dos questionamentos levantados e até dos sentimentos e anseios expressados por eles, emergiram discursos - analisados e organizados em categorias a seguir.

\section{Sexualidade e sua compreensão}

Diferentes conceitos sobre sexualidade são observados entre os adolescentes. As meninas, em sua maioria, relacionam a sexualidade ao desenvolvimento do corpo, ao namoro e às relações sexuais de modo mais afetivo. Já os meninos relacionam o sexo ao prazer, como se observa nos seguintes depoimentos:

"Eu sei que a sexualidade é quando o corpo muda, quando aparecem os seios e os pelos. É também a vontade de namorar, de beijar na boca, de conversar muito com o namorado e outras coisas estranhas que a gente sente." (MAS, menina)

"Pra mim, a sexualidade começa quando a gente menstrua pela primeira vez, ai, a gente não gosta mais de brincar de boneca e começa a conversar sobre namoro." (CRF, menina)

"Aprendi, aqui, no Peti que a sexualidade é quando duas pessoas que se gostam começam a namorar, pode ser um homem e uma mulher, ou dois homens, ou ainda duas mulheres." (PAL, menina)

"Sexualidade é a mesma coisa que querer fazer sexo com alguém." (MCF, menino)

"Pra mim a sexualidade é quando duas pessoas começam a namorar e querem fazer sexo." (OFS, menino)

As práticas e as representações dos sexos masculino e feminino não se mostram simétricas. No que diz respeito ao ato sexual, os homens o concebem como uma forma de

\section{Para o adolescente, o início da prática sexual traz uma série de dúvidas e tabus...}

dominação, de apropriação, de "posse" e as mulheres são socialmente preparadas para viver a sexualidade como uma experiência íntima e fortemente carregada de afetividade, que não inclui necessariamente a penetração, mas que pode englobar um amplo leque de atividades (falar, tocar, acariciar, abraçar etc. $)^{9}$.

A sexualidade é uma das dimensões do ser humano que combina gênero, identidade sexual, orientação sexual, erotismo, envolvimento emocional, amor e reprodução. É experimentada ou expressa em pensamentos, fantasias, desejos, crenças, atitudes, valores, atividades, práticas, papéis e relacionamentos ${ }^{10}$.

\section{Incertezas que envolvem a prática do sexo seguro e as DST}

Apesar dos profissionais do Peti desempenharem de modo responsável as ações de educação em saúde propostas, muitas foram as dúvidas e as informações incorretas citadas pelos adolescentes a respeito da prática do sexo seguro:

"Eu sei que a camisinha é o principal preservativo
contra as doenças causadas pelo sexo e para evitar
a gravidez. Eu até já vi uma camisinha, mas nem
sei como usar." (AJN, menino)
"Minha namorada usa umas pílulas que o posto de
saúde dá que protege pra não engravidar." (MFC,
menino)
"Se a camisinha previne a gravidez e as doenças,
por que a namorada do meu amigo ficou grávida,
já que ele falou que sempre usava a camisinha?"
(OFS, menino)

Para o adolescente, o início da prática sexual traz uma série de dúvidas e tabus que o acompanham e refletem na adoção, ou não, de medidas preventivas, sendo o uso correto do preservativo masculino uma ferramenta-chave na prevenção de problemas relacionados à saúde. 0 s adolescentes do sexo masculino apresentam limitados conhecimentos de manuseio desse recurso ${ }^{11}$. 
Assim, entende-se que as informações transmitidas a eles não são incorporadas de modo consistente ou o acesso a esse tipo de conhecimento se mostra deficiente.

\section{Dificuldades encontradas na educação sexual}

Muitos são os tabus que envolvem a sexualidade, principalmente quando a responsabilidade da educação sexual incide sobre os pais. Algumas falas ilustram a dificuldade de diálogo entre pais e filhos sobre o assunto:

"Na minha casa, não pode nem falar o nome sexo, porque minha mãe briga logo. Vejo é na TV como o casal faz sexo." (GSC, menino)

"Minha professora pede pra gente tirar todas as dúvidas que temos sobre a sexualidade, mas dá vergonha de perguntar, seus amigos ficam rindo de você... tenho medo de perguntar besteira." (RMT, menino)

"Lá em casa, eu nem gosto de falar sobre esses assuntos. Tiro minhas dúvidas é com minhas amigas." (MCF, menina)

Não há dúvida de que os primeiros educadores sexuais seriam os próprios pais, porque a eles compete a maior parcela de responsabilidade na formação dos filhos. Entretanto, como os pais, normalmente, têm dificuldades para falar sobre sexualidade com os filhos (na maioria dos casos de cunho cultural), essa tarefa foi deixada a cargo da escola ${ }^{12}$.

Outro aspecto relacionado é que, para lidar com a sexualidade dos filhos, os pais necessitam encarar sua própria sexualidade e tal situação pode gerar angústia. A sexualidade dos filhos traz à tona, para muitos pais, aspectos reprimidos de sua sexualidade ${ }^{13}$.

\section{Sociodrama e sua incorporação no aprendizado}

Depois da oficina de grupo focal, com vistas a atingir os significados manifestos sobre o ser adolescente diante da sociedade, recorreu-se à técnica sociodramática de Jacob Levy Moreno - com formação de 2 subgrupos, que realizaram o momento cênico com temas surgidos a partir de suas próprias dificuldades ressaltadas no momento do grupo focal:

"Ele só usa camisinha com uma garota séria, com outras nem liga"; e

"Meu filho se masturba todo dia."

No decorrer da dramatização do primeiro grupo, os participantes demonstraram a importância do uso da camisinha em todos os seus relacionamentos, independente

\section{A sexualidade dos filhos traz à tona, para muitos pais, aspectos reprimidos de sua sexualidade.}

do grau de afinidade ou estabilidade com o parceiro. Algumas falas emergiram durante a cena, como:

"A camisinha deve ser usada sempre que a gente fizer sexo com alguém, porque quem vê cara não vê doença." (MCF, menina)

"É preciso sempre procurar os profissionais da saúde, porque eles sabem informar bem e dão até o preservativo." (GSC, menino)

"O sexo só é seguro quando praticado com apenas um parceiro e usando os preservativos certos." (ARP, menina)

A aprendizagem pode ser definida como uma modificação, uma alteração do comportamento, comparando-se o antes e o depois de determinada situação. Isso geralmente reflete um aprimoramento de alguns tipos de performance - esta resulta do desenrolar de uma situação de aprendizagem ${ }^{14}$.

Os participantes do segundo grupo repassaram aos demais colegas a noção de que a masturbação constitui uma forma de conhecimento do próprio corpo, não sendo posta por eles como algo errado, que deve ser reprimido pelos pais:

"A masturbação não é uma doença, é uma necessidade do corpo." (LMS, menina)

"Devemos lembrar que orgasmo é um prazer que acontece depois de tocar nossos órgãos sexuais, $e$ só sentimos isso se a gente conhecer nosso corpo." (CRF, menina)

"Disse pra minha mãe que nessa fase da minha vida acontecem umas mudanças, aí, é bom eu conhecer meu corpo." (MLA, menino)

Desse modo, a dramatização como estratégia pedagógica possibilita o processo de ensino/aprendizagem centrado nos estudantes, tornando-os mais ativos, independentes, criativos, pensadores críticos, cooperativos, capazes de avaliar seus progressos - com desenvolvimento de boa capacidade de comunicação, bom relacionamento interpessoal, hábitos e técnicas adequadas para continuar 
aprendendo ao longo da vida ${ }^{15}$.

Vale destacar que muitos adolescentes afirmaram desejar que ocorram novos encontros com aplicação desse modelo metodológico, pois salientaram que tal estratégia proporcionou uma oportunidade de serem os próprios autores de suas histórias de vida.

\section{CONCLUSÃO}

Com as atividades realizadas, pôde-se observar que os adolescentes têm dúvidas e perguntas sobre esses assuntos que necessitam ser respondidas de modo franco e simples, uma vez que a temática se mostra difícil de ser explorada, principalmente pelo fato de que, hierarquicamente, ainda está ligada a uma complexa teia de medo, crenças e preconceitos.

Percebe-se que os momentos vivenciados contribuíram para o fortalecimento das relações interpessoais, que se mostraram fragilizadas no início dos encontros.

Portanto, abordar essa temática por meio de um mecanismo mais participativo, com técnicas grupais, possibilitou resgatar nos adolescentes a importância de sua atuação como agentes multiplicadores dessa proposta, enfatizando a necessidade de propagar a realização de atividades educativas como estratégia de socialização e de promoção da saúde no âmbito individual e coletivo.

Espera-se que este estudo possa contribuir para aumentar a potencialidade dessas práticas grupais, a fim de que sejam incorporadas como formas de cuidado e de estímulo à autonomia dos sujeitos envolvidos.

\section{CONTRIBUIÇÃO DAS AUTORAS}

Carla Patrícia Vieira de Miranda, Raquel Martins Mororó, Suyanne Maria Brito de Souza Rocha e Tiara Bruna Teixeira Teodósio contribuíram com a concepção, o delineamento e a realização da pesquisa. Maria Adelane Monteiro da Silva e Rebeca Sales Viana contribuíram com a análise dos dados e a redação e revisão crítica do manuscrito.

\section{REFERÊNCIAS}

1. Oliveira CD, Favere L. Conhecimento da sexualidade em um grupo de adolescentes. Boletim de Enfermagem. $2009 ; 2(3): 1-17$.

2. Sousa LT, Benicio AL, Santana MDR. Percepção de enfermeiros da Estratégia Saúde da Família em relação ao pré-natal com adolescentes. Sanare (Sobral, Online) [serial on the internet]. 2017 [cited 2017 Dec 12];16(1):39-44. Available from: https://sanare.emnuvens.com.br/sanare/ article/view/1092/603
3. Martins JJ, Albuquerque GL, Nascimento ERP, Barra DCC, Souza WGA, Pacheco WNS. Necessidades de educação em saúde dos cuidadores de pessoas idosas no domicílio. Texto \& Contexto Enferm [serial on the internet]. 2007 [cited 2017 Dec 12];16(2):254-62. Available from: Available from: http://www.scielo.br/pdf/tce/v16n2/a07v16n2.pdf

4. Brasil. Ministério do Desenvolvimento Social [document on the internet]. 2004 [cited 2013 Nov 22]. Available from: www.mds.gov.br

5. Minayo MCS. 0 desafio do conhecimento: pesquisa qualitativa em saúde. 4. ed. São Paulo: Hucitec; 1996.

6. Queiroz DT, Vall J, Vieira NFC. Observação participante na pesquisa qualitativa: conceitos e aplicações na área da saúde. Rev Enferm UERJ [serial on the internet]. 2007 [cited 2017 Dec 12];15(2):276-83. Available from: http://www. facenf.uerj.br/v15n2/v15n2a19.pdf

7. Backes DS, Colomé JS, Erdmann RH, Lunard VL. Grupo focal como técnica de coleta e análise de dados em pesquisas qualitativas. Mundo Saúde [serial on the internet]. 2011 [cited 2017 Dec 12];35(4):438-42. Available from: http:// bvsms.saude.gov.br/bvs/artigos/grupo focal_como_tecnica coleta analise dados pesquisa qualitativa.pdf

8. Moreno JL. Psicoterapia de grupo e psicodrama. São Paulo: Mestre Jou; 1959.

9. Bourdieu P. A dominação masculina. Rio de Janeiro: Bertrand Brasil; 1999.

10. Castro MG, Abramovay M, Silva LB. Juventude e sexualidade. Brasília (DF): Unesco; 2004.

11. Crosby RA, Sanders SA, Yarber WL, Graham CA. Condom use errors and problems: a neglected aspect of studies assessing condom effectiveness. Am J Prev Med. 2003;24(4):367-70.

12. Azevedo MPS. Educação sexual ou orientação sexual? [document on the internet]. 2001 [cited 2013 Nov 19]. Available from: http://www.tvebrasil.com.br/SALTO/ zoletins2001/se2/se2txt1.htm

13. Medeiros M, Ferriani MGC, Munari DB, Gomes R. A sexualidade para o adolescente em situação de rua em Goiânia. Rev Latinoam Enferm [serial on the internet]. 2001 [cited 2017 Dec 12];9(2):35-41. Available from: http://www. scielo.br/pdf/rlae/v9n2/11512.pdf

14. Alcântara C. Psiquê e sexualidade [document on the internet]. 2010 [cited 2013 Nov 19]. Available from: http:// www.psiqueesexualidade.com/2010/01/sobre-aprendizagem. $\underline{\text { html }}$

15. Silva EL, Menezes EM. Metodologia da pesquisa e elaboração de dissertação. 4. ed. Florianópolis: Universidade Federal de Santa Catarina; 2005.

Recebido em 16/05/2017 Aprovado em 15/11/2017

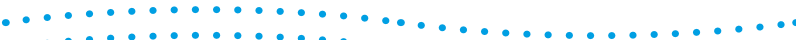
$\ldots$ $\ldots \ldots \ldots \ldots$ 